Recuperación de trabajo de fin de grado

\title{
Una filosofía de la práctica desde el peronismo
}

\section{(Acción y pensamiento de John William Cooke entre 1945 y 1959)}

A philosophy of praxis from Peronism (Action and thought of John William Cooke between 1945 and 1959)

Rafael Zamarguilea

Licenciado en Ciencia Política

(Universidad Nacional de Rosario)

Subsecretario de Extensión de la

Facultad de Ciencias Médicas

(Universidad Nacional de Rosario)

Correo: rafaelzamarguilea@gmail.com 


\title{
Resumen
}

El presente artículo tiene por finalidad exponer resumidamente los nudos fundamentales del abordaje de la obra de John William Cooke (1919-1968) realizado en la tesina de grado dirigida por el profesor Sebastián Artola y presentada en junio 2019 como requisito último para la obtención el título de Lic. en Ciencia Política. El trabajo propone analizar el peronismo de Cooke desde una perspectiva de traducibilidad de los lenguajes políticos y filosóficos que contribuya a su valoración en tanto filosofía de la praxis, en diálogo y polémica con otros enfoques al respecto. Para eso, se lleva adelante una lectura contextual de sus principales escritos entre los años 1945 y 1959, apuntando a una comprensión que recupere la intención compleja del autor y reconstruya la historicidad de los debates en que se encontraba inscripta. De este modo, se aportan elementos críticos respecto de las interpretaciones más unilaterales de la biografía intelectual de Cooke y se reactualizan algunos de sus principales aportes a la teoría política a partir del reconocimiento de las tensiones tramadas en su obra, enmarcada tanto en el peronismo como en el marxismo.

Palabras clave

Cooke, Peronismo, Marxismo, Insurrección.

\begin{abstract}
The purpose of this article is to summarize the main points of the approach to the work of John William Cooke (1919-1968) carried out in the research directed by Professor Sebastián Artola and presented in June 2019 as a final requirement for obtaining the degree in Political Science. The work proposes to analyze Cooke's Peronism from a perspective of translatability of political and philosophical languages that contributes to its assessment as a philosophy of praxis, in dialogue and polemic with other approaches in this regard. To this end, a contextual reading of his main writings between 1945 and 1959 is carried out, aiming at an understanding that recovers the author's complex intention and reconstructs the historicity of the debates in which it was inscribed. In this way, critical elements are provided with respect to the most unilateral interpretations of Cooke's intellectual biography and some of his main contributions to political theory are updated from the recognition of the tensions plotted in his work, framed both in Peronism and Marxism.
\end{abstract}

Keywords

Cooke, Peronism, Marxism, Insurrection. 


\section{Introducción}

En un pequeño artículo sobre Cooke, Horacio González menciona que "toda la política argentina medida frente a Cooke muestra su carácter incompleto y desdichado" (1999:9). Enunciación que refleja en gran medida las impresiones que me llevaron de indagar en la obra y biografía de quien fuera uno de los protagonistas más destacados de la política argentina del siglo XX.

A diferencia de múltiples autores y autoras del campo nacional-popular y de la izquierda de la Argentina, John William Cooke tiene la particularidad de haberse representado siempre como un político antes que como un intelectual. Efectivamente, puede afirmarse que fue un político de primera línea, con la responsabilidad de conducir un movimiento de masas como el de la resistencia peronista entre 1956 y 1959. Y desde allí partieron generalmente sus elaboraciones teóricas. Sin embargo, resulta notable que, en muchas ocasiones, se lo haya estudiado de un modo impropio para dicha especificidad: disolviendo la riqueza de su vida política concreta en una hermenéutica sobre sus textos centrada en aquellos aspectos ideológicos más estereotipados. Los cuales, según el canon de cada exegeta, ubican a Cooke -o alguno de los momentos de su trayectoria- dentro de una determinada corriente teórico-política marxista, peronista o nacionalista. Por el contrario, nuestra perspectiva no apunta sólo hacia el examen de tal o cual referencia más o menos explícita a la lucha de clases o a la comunidad organizada, sino que pretende comprender la intención compleja de sus enunciados, a través de un análisis contextual y crítico que establezca una relación de traducibilidad entre los diferentes lenguajes políticos habitados por el autor.

Nos preguntamos, en este sentido, sobre pertinencia de ponderar estos enunciados en tanto momentos del desarrollo de un pensamiento-acción entendido como proceso y como parte del desenvolvimiento de una filosofía que es también una política, ligada orgánica y pedagógicamente a la lucha revolucionaria de la clase trabajadora argentina, es decir, en términos de Gramsci (1970): una filosofía de la praxis. En ese caso, la tan referida contraposición entre peronismo y marxismo podría ser re-semantizada, al menos para el caso de Cooke, de un modo que permita entender sus aportes a la 
teoría de la revolución en la Argentina, hechos desde el seno del peronismo, como la avanzada de una renovación del marxismo argentino en momentos en que su pretendida vanguardia, el Partido Comunista Argentino, padecía de una alienación ideológica patente, luego de haberse ubicado detrás de la Unión Democrática y en contra de la movilización obrera que confluyó en el nacimiento del movimiento peronista.

La carencia de una síntesis teórico-política propia, específicamente nacional y latinoamericana, se convirtió a partir de entonces en un drama para las corrientes populares y antiimperialistas de la Argentina que tuvo en Cooke a uno de sus exhortadores más tempraneros. De esto dan cuenta textos notables de su autoría de la década del sesenta, como Aportes para una crítica del reformismo en la Argentina (1961), Informe a las bases (1966) o La revolución y el peronismo (1967).

Sin embargo, no son estos escritos el foco de la presente investigación, sino la trayectoria de Cooke entre 1945, año de su incorporación al emergente movimiento peronista, y 1959, año de su definitivo desplazamiento de la conducción de la resistencia peronista. Se recorren, entre tanto, sus años como diputado nacional entre 1946 y 1952, su desempeño desde llano entre 1953 y 1955, y su ulterior encumbramiento como primer delegado de Perón en el exilio a partir de 1956.

De este modo, en sintonía con el propósito de aportar a la comprensión de la obra Cooke desde una perspectiva capaz de vincular orgánica y dialécticamente la teoría y la práctica, nuestro recorte apunta a su etapa de mayor ascendencia real sobre la política, tanto del movimiento peronista como del movimiento obrero.

Traducibilidad de los lenguajes filosóficos y contextualización de los enunciados políticos

Las características del objeto de estudio y la complejidad del problema planteado implican un acercamiento desde distintas dimensiones metodológicas. Por un lado, resultan pertinentes algunas de las precauciones planteadas por la escuela de Cambridge apuntadas a evitar el "riesgo de caer en varios tipos de absurdo histórico" y llegar a resultados que puedan ser 
calificados de "mitologías" antes que "historias" (Skinner, 2000:152). Sobre la base de reconocer las diferencias significativas que median entre su objeto de estudio y el nuestro, el rescate de este enfoque se centra a la idea de recuperar la "intención compleja de autor" (2000:188) como condición habilitante de un diálogo productivo entre discusión filosófica y evidencia histórica. Se pretende así confrontar las diferentes interpretaciones de los textos cookistas con sus verdaderas condiciones de posibilidad, debatiendo con aquellas recuperaciones más tendencializadas que encuentran un Cooke funcional a sus propias necesidades teórico-políticas a costa de sacrificar el reconocimiento de su complejidad.

Debe entenderse que, si bien el contexto no explica de por sí las posiciones de un autor, permite descartar anacronismos y replantear interpretaciones unilaterales, como las que omiten el hecho de que cualquier definición de tipo ideológica constituía para Cooke un problema práctico antes que teórico, en la medida en que afectaba su capacidad de liderazgo, así como las perspectivas del movimiento.

Por otro lado, abordar la obra Cooke implica trabajar sobre el cruce de diferentes lenguajes políticos, fundamentalmente, peronismo y marxismo. Los cuales traman una particular tensión que recorre el conjunto de su obra y que, de algún modo, la define. Se torna indispensable, en este sentido, establecer una relación de traducibilidad, retomando la noción de "traducibilidad de los lenguajes científicos y filosóficos” de Gramsci (1971:72). Esto implica quitar el foco de lo doctrinario para ponerlo en la acción concreta y en la práctica histórica, entendiendo al marxismo, en tanto filosofía de la praxis, como una nueva inmanencia capaz establecer una relación de traducibilidad, orgánica y profunda, polémica y abierta con todas las teorías y visiones existentes (Dal Maso, 2016). La consecuencia metodológica de estos postulados es que la filosofía de un hombre de acción deba buscarse en sus textos políticos antes que filosóficos. Los cuales deben ser contextualizados a los fines de ubicar las particulares articulaciones teórico-políticas subyacentes, vinculando dialécticamente aspectos históricos, filosóficos, políticos y económicos, generales y particulares, que hacen a la obra. 
Por último, resulta clave indagar en los distintos modos en que el legado de Cooke persiste a través de las apropiaciones de diferentes corrientes y ensayistas. De allí que primeramente se haga eje en los diferentes estudios biográficos y críticos sobre Cooke, poniéndolos en diálogo para, de este modo, examinarlos críticamente.

Biografía intelectual y obra de John William Cooke

Al hacer un repaso por la bibliografía sobre Cooke puede apreciarse que, pese a no ocupar el lugar que corresponde a la magnitud de su obra, esta ha sido recuperada con el transcurrir de los años por una cantidad de investigaciones históricas y biografías intelectuales y políticas. Lo que permite, junto con la publicación de sus Obras completas, compiladas en cinco tomos por Eduardo Luis Duhalde entre 2008 y 2011, un acceso bastante completo a su trayectoria, así como a diferentes perspectivas desde las cuales interpretarla.

Así pues, se registran como primeros antecedentes a los trabajos de Ernesto Goldar (1985), Cooke y peronismo revolucionario, y Horacio González (1986), La revolución en tinta limón. Recordando a John William Cooke, pioneros en la recuperación del legado cookista. La primera investigación completa de la biografía de Cooke corresponde, sin embargo, al historiador británico Richard Gillespie (1989): J. W. Cooke, el peronismo alternativo; incorporándose luego Cooke, de Perón al Che de Norberto Galasso (1997), John William Cooke. La mano izquierda de Perón de Daniel Sorín (2014), y El hereje. Apuntes sobre John William Cooke de Miguel Mazzeo (2016). Se analiza también El peronismo republicano. John William Cooke en el Parlamento Nacional, de Cristian Gaude (2014), que aborda específicamente las concepciones políticas implícitas en la labor de Cooke dentro de la Cámara de Diputados. A modo de síntesis, puede decirse que estos trabajos se debaten entre una visión más etapista o evolucionista del recorrido vital de Cooke y otra que pondera las continuidades existentes en cuanto a su compromiso antiimperialista y popular.

Además, se recuperan críticamente las perspectivas presentadas en la novela filosófica La astucia de la razón, de José Pablo Feinmann (2014), y en el ensayo sobre el pensamiento argentino Restos Pampeanos, de Horacio Gonzá- 
lez (2007). Estos escritos, si bien no remiten exclusivamente al pensamiento de Cooke, le otorgan un lugar preponderante y exponen dos maneras divergentes de comprenderlo que resultan paradigmáticas. Para el primero, Cooke representa la expresión más acabada del pensamiento centrista, es decir, de la necesidad de que el marxismo y la izquierda revolucionaria argentina se incorporen al movimiento peronista como único modo de hacer pie entre la clase trabajadora. Mientras que, para el segundo, la noción del peronismo como "hecho maldito del país burgués" apunta también a los costos de esa pertenencia, en tanto constituye una afirmación de Cooke respecto de las características revolucionarias del peronismo lo mismo que una denuncia de su carácter burocrático, reformista y hasta retardatario.

También se consideran cruciales textos sobre la historia del peronismo como los de Alejandro Horowiczs (2011), Daniel James (2010) y Claudio Spiguel (2016). Autores que aportan una contextualización que lejos de objetivar al peronismo como "fenómeno", como algo irracional o como una anomalía -como pretende cierta perspectiva cientificista de la historia (Pigna, 2015)-, apuntan a comprenderlo en tanto sujeto político de un drama histórico.

Son los escritos de Cooke, finalmente, los que aportan las herramientas insustituibles a la hora comprender el carácter complejo de las relaciones que establecieron peronismo, clase trabajadora y marxismo. Se trata de una obra copiosa y, al mismo tiempo, fragmentaria, compuesta por apuntes, proyectos, conferencias, entrevistas, informes, artículos y cartas que no necesariamente concibieron para su publicación. Razón por la cual, para su comprensión, resulta indispensable ponderar la coyuntura política en que cada enunciado fue concebido y fundamentalmente el espacio político concreto que su autor ocupaba en ese momento, para así poder interpretarlos correctamente, teniendo en cuenta a quiénes iba dirigido y también contra qué y quiénes apuntaba.

De este modo, nuestro trabajo aborda el análisis exhaustivo de los escritos contenidos en los tomos I, II y IV de las Obras Completas de Cooke (2008a, 2008b, 2010). Destacándose, dentro del periodo parlamentario, el análisis de la conferencia Perspectivas de una economía nacional de 1948, del an- 
teproyecto de reforma constitucional y de sus discursos parlamentarios respecto de la ley de Represión a los Actos de Monopolio, las Actas de Chapultepec, la nacionalización de depósitos bancarios, la expropiación del diario La prensa y el fallido golpe de 1951.

$\mathrm{Al}$ respecto, debe resaltarse que al enfocar el análisis de la obra de Cooke al trasluz de su cronología vital puede evidenciarse su carácter de proceso, que no necesariamente quiere decir evolución, concepto que muchos autores asocian -con cierta razón- a una subestimación del peronismo como concepción política. Puede decirse, no obstante, que Cooke pasó de un antiimperialismo democrático y popular fuertemente inspirado por el liderazgo de Perón y su doctrina de la comunidad organizada a una visión cada vez más autocrítica de las limitaciones peronismo, primero en relación a cuestiones tácticas y posteriormente también estratégicas.

A saber, debe mencionarse que la insurrección ocupó un lugar en sus reflexiones ya desde el histórico 17 de octubre de 1945. Y si bien el cambio de situación que implicó la llegada de Perón a la presidencia mediante el voto popular, y el ulterior rol parlamentario que asumió Cooke, determinaron que los temas prioritarios sean otros, cuando los vientos empezaron a virar, no dudó en remarcar frente al hemiciclo que el peronismo no era un simple partido político sino una revolución y que "tanto por la vía de las armas como por la vía de comicios estamos dispuestos a enfrentar” (Cooke, 2008a:421).

Del periodo que va entre la finalización de su mandato como diputado y la caída del gobierno de Perón se consideran fundamentalmente algunas de las editoriales de la revista De Frente, referidas a debates públicos como los del Congreso de la Productividad, el conflicto con la Iglesia y el acuerdo petrolero con la California. También los artículos sobre la situación internacional referidos a la caída de Getulio Vargas en Brasil y Jacobo Árbenz en Guatemala, donde Cooke profundiza su mirada sobre la necesidad de "desmontar la máquina que ha servido para oprimir al país" (Cooke, 2010:62).

Del período entre los años 1956 y 1958, en los que Cooke se desempeñó como delegado de Perón, se analiza la correspondencia con este y especialmente el Informe Generaly Plan de Acción posterior a las elecciones de convencionales constituyentes de 1957. Por último, se aborda la conferencia 
pronunciada en el Congreso de la Liberación Nacional de octubre de 1959, titulada La Lucha por Liberación Nacional, en donde Cooke, ya des-promovido de la dirección del movimiento, pero aun sin haberse vinculado a Cuba, plantea una plataforma política revolucionaria de carácter integral que lo aleja definitivamente del marco estratégico propiciado por Perón.

La pregunta que sobrevuela en "La lucha por la liberación nacional" es por qué, lejos de consolidarse como una unidad combatiente, a medida que la represión amainaba y la Liberadora abría espacios de semi legalidad, volvieron a aflorar en el peronismo la ambigüedad, el burocratismo, la indisciplina, el oportunismo, el electoralismo y la mezquina disputa de intereses a espaldas de la masa, que lo habían llevado a la derrota en 1955. Cuestión que Cooke había advertido ya en el Informe y Plan de Acción, planteándolo incluso como una cuestión de clases sociales. Allí, no sólo se reconocía que el liderazgo de Perón y su mito propiciaban la convergencia de una variedad de posturas ideológicas que naturalmente tendían al conflicto, sino que se postulaba que el peronismo exhibía - tal como la dialéctica hegeliana para Marx- algo así un núcleo racional contrapuesto a su envoltura mística: por un lado, las masas obreras que protagonizaron el 17 de octubre y, por el otro, una dirigencia con la que no podía contarse para las tareas insurreccionales:

Mientras la clase obrera tomó conciencia inmediata de los valores del peronismo como Movimiento Nacional-Libertador Revolucionario, parte de la burguesía siguió operando con los viejos conceptos del pasado político argentino. La parte más avanzada piensa en términos de Yrigoyenismo; sin comprender que Yrigoyen fue el último gran político del pasado, iniciador de una nueva época, de cuyo estilo no podrá apartarse ya la línea del 'desenvolvimiento nacional'. De forma que son progresistas con relación a una época ya perimida, pero reaccionarios con relación a las nuevas formas que toma la lucha por el poder social en la argentina (Cooke, 2008b:265).

Es importante remarcar que el Informe mantiene su fidelidad al planteo de la comunidad organizada, al menos en última instancia, pero proponiendo que la clase obrera se constituya en dirección efectiva del movimiento, es decir, invirtiendo los términos en que esta se jerarquizaba. Así, si la línea insurreccional había sido una respuesta en principio táctica a la disgregación 
del peronismo, ahora debía tornarse estrategia, orientando no solo la organización clandestina y la lucha sindical, sino la propia lucha política, que ya no debía delegarse en la vieja burocracia partidaria. Una política insurreccional implicaba, en este sentido, la integración del movimiento político y el gremial:

En la acción se sella la solidaridad política del todo porque se politizan las luchas gremiales, lo que equivale a realizar y llevar a la práctica una política insurreccional, sin la cual la insurrección, como culminación de un proceso, no es concebible, ya que solo por razones políticas el pueblo en su conjunto considera que la toma del poder es la única garantía para satisfacer sus apetencias económicas y sociales (Cooke, 2008b:275).

Es necesario destacar que "La lucha por la liberación nacional" constituye, antes que nada, un análisis del espontaneísmo de la resistencia, de sus potencialidades, sus limitaciones, su irrupción, su derrota y las posibilidades de revertirla. Y que sus conclusiones fueron elaboradas incluso antes de que la Revolución Cubana consolide su perfil y formule teóricamente la cuestión de la impotencia de las burguesías nacionales latinoamericanas, en la Segunda Declaración de La Habana de 1962. Esto quiere decir que, mientras la mayor parte del peronismo seguía preso de la ilusión por reeditar el frente del 45 y la izquierda argentina se debatía entre la teoría de la revolución democrático-burguesa y la teoría de la revolución socialista de inicio, Cooke señalaba el camino de una revolución popular y democrática, con tareas agrarias y nacionales pero hegemonizada por la clase obrera.

Los terratenientes dependen de los intereses de Gran Bretaña. La burguesía industrial, en su mayor parte está subordinada o deseando subordinarse al imperialismo y se apoya en él para acentuar su predominio interno. Como clase carece de empuje, y lejos de afirmarse como clase nacional -para lo cual contó con el impulso que dio el gobierno de Perón al desarrollo industrialpactó sistemáticamente con la oligarquía vacuna y con las fuerzas colonialistas. La liberación nacional y la revolución social no son dos asuntos independientes o paralelos, sino un solo problema indivisible (Cooke, 2010:132). 
De este modo, lo que el Informe había adelantado desde una perspectiva predominantemente práctica es apuntalado en "La Lucha por la liberación nacional" mediante una valorización explícita de la teoría marxista que, aunque no de forma lineal, se consolida en textos posteriores:

$\mathrm{Al}$ analizar el papel de la clase trabajadora en el Frente de Liberación, debe partirse del hecho concreto de que la lucha de clases existe y no se trata, como sostiene la reacción, de un invento comunista. El marxismo ha analizado el problema, pero no lo ha creado, porque la lucha de clases no es una teoría, sino un hecho (...) querer solucionar los problemas de ella derivados por medio de fórmulas conciliadoras es creer en la magia negra y ser tan reaccionario como los que niegan su existencia (Cooke, 2010:132).

\section{Conclusiones}

En suma, al ligar la cuestión nacional a la lucha de clases, Cooke avanza en 1959 hacia un planteo de carácter socialista que termina de explicitar durante su exilio en Cuba. Pero, a diferencia de quienes suponen que Cuba llevó a un cambio radical en su pensamiento, el presente trabajo comprende que la vivencia de la Revolución Cubana representó tanto un descubrimiento como una confirmación de aquello que venía trazando desde su propia práctica al frente de la resistencia peronista.

Como señala Miguel Mazzeo (2016), es evidente que Cooke dejó de reconocer a Perón como su referente estratégico al menos a partir de 1959. Su relación con el líder exiliado quedó determinada a partir de entonces por la perspectiva de que la dinámica de las luchas populares lo incline hacia un cambio estratégico. Y su posterior encuentro con el Che Guevara, en este sentido, lejos de constituir una casualidad, debe pensarse como inscripto en esta dinámica.

Así pues, puede concluirse que las tesituras de Cooke, antes que un elemento exógeno a la realidad argentina y, específicamente, a la realidad de la resistencia peronista y el movimiento obrero, constituyeron verdaderas lecciones de una derrota que lamentablemente no llegó a ver revertida, al morir prematuramente en 1968, un año antes de que la clase obrera y el 
movimiento estudiantil desaten en la Argentina un nuevo auge de luchas populares de proporciones históricas.

\section{Referencias bibliográficas}

Cooke, John William (2008a). Obras completas, tomo I: Acción parlamentaria (Eduardo L. Duhalde comp.). Buenos Aires, Argentina, Colihue.

Cooke, John William (2008b). Obras completas, tomo II: Correspondencia Perón-Cooke (Eduardo L. Duhalde comp.). Buenos Aires, Argentina, Colihue.

Cooke, John William (2009). Obras completas, tomo III: Articulos periodísticos, reportajes y cartas (Eduardo L. Duhalde comp.). Buenos Aires, Argentina, Colihue.

Cooke, John William (2010). Obras completas, tomo IV: Artículos periodísticos, reportajes y cartas (1947-1959) (Eduardo L. Duhalde comp.). Buenos Aires, Argentina, Colihue.

Cooke, John William (2011). Obras completas, tomo V: Peronismo y revolución (Eduardo L. Dubalde comp.). Buenos Aires, Argentina, Colihue.

Dal Maso, Juan (2016). El marxismo de Gramsci: notas de lectura sobre los Cuadernos de lectura. Buenos Aires, Argentina, Ediciones IPS.

Feinmann, José Pablo (2014). La astucia de la razón. Buenos Aires, Argentina, Planeta.

Galasso, Norberto (2004). Cooke: de Perón al Ché. Buenos Aires, Argentina, Nuevos Tiempos.

Gaude, Cristian. (2014). El peronismo republicano. John William Cooke en el Parlamento Nacional (Tesis de grado). Universidad Nacional de General Sarmiento, Los Polvorines.

Gillespie, Richard (1989). J.W. Cooke. El peronismo alternativo. Buenos Aires, Argentina, Cántaro.

González, Horacio (1999). “Cooke, el cincel de una derrota”. En: M. Mazzeo (Ed.) Cooke, de vuelta (El gran descartado de la bistoria argentina) (pp. 7-10). Buenos Aires, La Rosa Blindada.

González, Horacio (2007). Restos pampeanos. Buenos Aires, Argentina, Colihue.

Gramsci, Antonio (1970). Introducción a la filosofía de la praxis. Barcelona, España, Ediciones Península.

Gramsci, Antonio (1971). El materialismo histórico y la filosofía de Benedetto Croce. Buenos Aires, Argenrina, Nueva Visión.

Horowicz, Alejandro (2011). Los cuatro peronismos. Buenos Aires, Argentina, Edhasa.

James, Daniel (2010). Resistencia e integración. Buenos Aires, Argentina, Siglo XXI.

Mazzeo, Miguel (1999). Cooke, de vuelta (el gran descartado de la historia). Buenos Aires, Argentina, La Rosa Blindada.

Mazzeo, Miguel (2000). John William Cooke. Textos Traspapelados (1957-1961). Buenos Aires, Argentina, La Rosa Blindad.

Mazzeo, Miguel (2016). El Hereje. Apuntes sobre John William Cooke. Buenos Aires, Argentina, El Colectivo. 
Pigna, Felipe (2015). "Dos, tres, cuatro, muchos peronismos”. En A. Horowicz (Ed.), Qué queda de los cuatro peronismos (pp. 131-149). Buenos Aires, Argentina, Octubre.

Skinner, Quentin (2002). Significado y comprensión en historia de las ideas. Prismas, 4(4), 149-191.

Sorín, Daniel (2014). John William Cooke. La mano izquierda de Perón. Buenos Aires, Argentina, Planeta.

Spiguel, Claudio (2016). "La clase obrera argentina y el peronismo histórico". En C. Mateu y C. Spiguel (Eds.). Movimiento Obrero Argentino. Aspectos y momentos históricos de la lucha política y sindical (pp. 50-88). Buenos Aires, Argentina, Revista La Marea.

Aceptado: 9/02/2021

Recibido: 16/04/2021 